\title{
Factual Quantification Methods of Energy Consumption for Transmission Nodes in Cellular Communication Systems : Experimental case study in Lome
}

Kodjovi Semenyo Agbosse ], Koffi Agbeblewu Dotche 2 [https://orcid.org/0000-0002-5945-0036], Adekunlé Akim Salami ${ }^{3}$ [https://orcid.org/0000-0002-0917-1231] , Koffi Mawugno Kodjo 4 [ https://orcid.org/0000-0001-5674-2212] and Ayité Sénah Akoda Ajavon 5 [https://orcid.org/0000-0002-6318-2396]

1, 2, 3,4, 5 Department of Electrical Engineering, CERME (ENSI), University of Lome, Lomé- Togo

${ }^{2}$ Department of Electrical Technology, Akenten Appiah Menka-University of Skills Training and Entrepreneurial Development (AAMUSTED), Kumasi-Ghana

\begin{abstract}
The energy efficiency for wireless communication technology standard is very important for the current and the future generation ones. Noting that the energy consumption in communication systems is constantly increasing due to the exponential number of subscribers and high data services demand. In this regard, it becomes necessary to quantify this energy consumption with respect to the communication technology standard at the site. This article presents an evaluation of the factual quantifications methods for the energy consumption of transmission nodes in cellular communications systems. The data collection was obtained on three types of communication technology standards namely second generation (2G), 3G, 4G and their combination using the direct and indirect (the utility records) methods power measurement on the field installation of a mobile telephone operator in Togo. These data have undergone a preprocessing in the Microsoft Excel software (version 2019), then sent in the Matlab software (Matlab R2020b) for further analysis. The results showed that the energy consumption observed at the site is around $124 \mathrm{kWh}, 254$, $\mathrm{kWh}$ and $362 \mathrm{kWh}$ on monthly average respectively for $2 \mathrm{G}, 2 \mathrm{G} / 3 \mathrm{G}$, and 2G/3G/4G typology used. It further indicated that when more communication standards are used on a given site, its power consumption is much more increasing. The power profile distribution has been investigated, and the analysis revealed that the normal distribution closely fitted the data. However, more parameters related to the number of utilised channels and climatic conditions need to be considered in future research works.
\end{abstract}

Keywords: cellular communication systems, distribution fitting tool, energy consumption, power profile quantification

\section{Introduction}

Please notice The cellular networks rely on the efficient use of the spectrum therefore the service area may be divided into smaller cells, ideally with no gaps or overlaps. Each cell, is served by its own base station and a set of frequencies. The goal behind this concept was to increase capacity so the network can provide service to a large number of subscribers while maintaining optimal system performance. The Groupe Spéciale Mobile acronym GSM was first used in 1982 by the European Standardization organization, the task group (GSM) was mandated to define a new standard of mobile communication in the band of $900 \mathrm{MHz}$. This name was changed to Global System for Mobile Communication (GSM) in 1989 and has since remained GSM's name. The air interface of GSM is a combination of Time Division 
Multiple Access and Frequency Division Multiple Access [1]. GSM is known as secondgeneration network. GSM technology has some limitations in high data application and bandwidth efficiency. This is explained by the introduction of the Code Division Multiple Access (CDMA). The CDMA is an alternative to GSM, because of the delivery of high-speed data packets such as multimedia, video, call conference etc., as required for the Third Generation (3G) networks. The greatest advantage of CDMA compared to other wireless cellular systems was the potentially enhanced voice capacity and its ability to work effectively in noisy environments.

The name of the access network is related to the generation of the cellular system, subsequently Network Sub-System (NSS) for 2G, Universal Terrestrial Access Network (UTRAN) for 3G. A 4G evolved-UTRAN (eUTRAN) has a simplified architecture (compared to a 3G-UTRAN), which integrates the eNode B base stations' control functions that were previously implemented in base station controllers called RNC (Radio Network Controller of $3 G$ ) and Base Station Controllers (BSC) of RAN (Radio Access Network) networks. The main improvements of LTE-Advanced (LTE-A) residing in the 4G mobile network standard [2] are :

- carrier aggregation : consists of serving a user on several carriers at the same time, this allows to obtain a bandwidth of about $100 \mathrm{MHz}$ (aggregation of five carriers of 20 $\mathrm{MHz}$ bandwidth) while one can achieve a 1 Gbits / s downstream speed;

- support for a heterogeneous network: this is an important advantage of LTE-A, which consists in deploying low-power stations (pico, femto, relay) in macro cells; this improves the quality of service (speed in particular) in areas with high population density or poorly covered;

- multiple antenna use: LTE-A and future mobile communications networks use a transmission mode that supports downlink MIMO configuration.

It will be recalled that the traditional macrocell base stations do consume some significant amount of energy at the network access due to the power amplifier low efficiency. The evolution to subsequent technology aims at reducing this energy consumption by introducing natural cooling. In regard to the sustainable technological development and free emission of dioxide of carbon policy, the renewable energy to supply the transmission nodes are gained much interest in the scientific community [3]. Thus, in practical network, different typologies are used at a cell site depending on the geographical area. It becomes necessary therefore to quantify the energy consumption by these typologies with respect to the communication technology standards that are used at the site. This article presents an evaluation of the factual quantifications methods for the energy consumption of transmission nodes in cellular communications systems.

The rest of this paper is structured as follows, the section 2 presents the theory behind the power modelling of a cluster-group of transmitters. In section 3 , the methodology of the data collection is given and the results are in section 4 . The last section is the conclusion.

\section{The Model}

The linear model of power consumption $P_{i, k}$, at a transmission node [3] is given as follows :

$$
P_{i, k}=\frac{1}{\eta} P_{t, i}+\alpha P_{c}+P_{0}
$$

$\eta$ is the efficiency of power amplifier, $p_{t}$, the transmit power of the node, $\alpha$ the number of antennas at the node, the circuit power $P_{c}$, and $P_{0}$ is the non-transmission power consumption including $P_{D P}$ baseband signal processing, $P_{C o n v}$, AC-DC converter ; $P_{C o o l}$, cooling ; $P_{L B}$, the lighting system; $E_{b b}$, battery backup (uncharged), etc...

The non-transmission power, $P_{0}$, is given by 


$$
P_{0}=P_{D P}+P_{C o n v}+\sum_{i}^{m} P_{C o o l}+\sum_{j}^{n} P_{L B_{j}}+E_{b b}+P_{\text {link }}
$$

Thus for a cluster group of base station the total power consumption, $P_{T o}$ of the system is given as:

$$
P_{T o}=\sum_{k=1}^{K} n_{k} \cdot P_{k}+P_{B a c h}
$$

where $\mathrm{K}$ is the number of base station types used in the network, $n_{k}$ is the total number of base stations of a specific type k-th (e.g., Macro base stations), $P_{k}$ is the power consumption of a base station of type $\mathrm{k}$, and $P_{B a c h}$ is the backhaul power loss [4].

\section{Methodology}

This section presents the data collection, sampling and processing steps

\section{Data Collection}

The power measurements were conducted on Moov-Africa cellular network's installation in Togo. The power level against the top-level KPIs obtained by a Nokia Siemens Network Statistics tool.

\section{Sample size and data processing}

The data sampling size has consisted in the averaging of the raw data over a monthly basis. That implied that, the arithmetic average values were considered.

The measured power data's collection spans over a 9 month, and has considered the following observation time intervals :

- hour: the hourly statistics gives a detailed picture of the network performance and are useful to help spot temporary problems and identify trends.

- peak or busy hour : Peak hour statistics are of great significance because they correspond to the time of heavy utilization of network resources. In a way, they provide the "worst-case" scenario.

- day: daily statistics are introduced to provide a way of averaging temporary fluctuations of hourly data.

The data were processed into excel spreadsheet, and saved as a cascaded sheet .CSV file before been processed into the Matlab software (Matlab R2020b) where the distribution fitting tool (dftool) was used for further analysis to help in quantifying the power profile distribution observed.

\section{Results and discussion}

In this section the results are presented and discussed.

In Figure 1, it shows that the power consumption based on the typologies of technology used on given a site may subsequently vary. It may however be stated when the three typologies are been deployed the consumption could be high. This high value may strongly depend on the geographical area by then on the subscribers' density. 


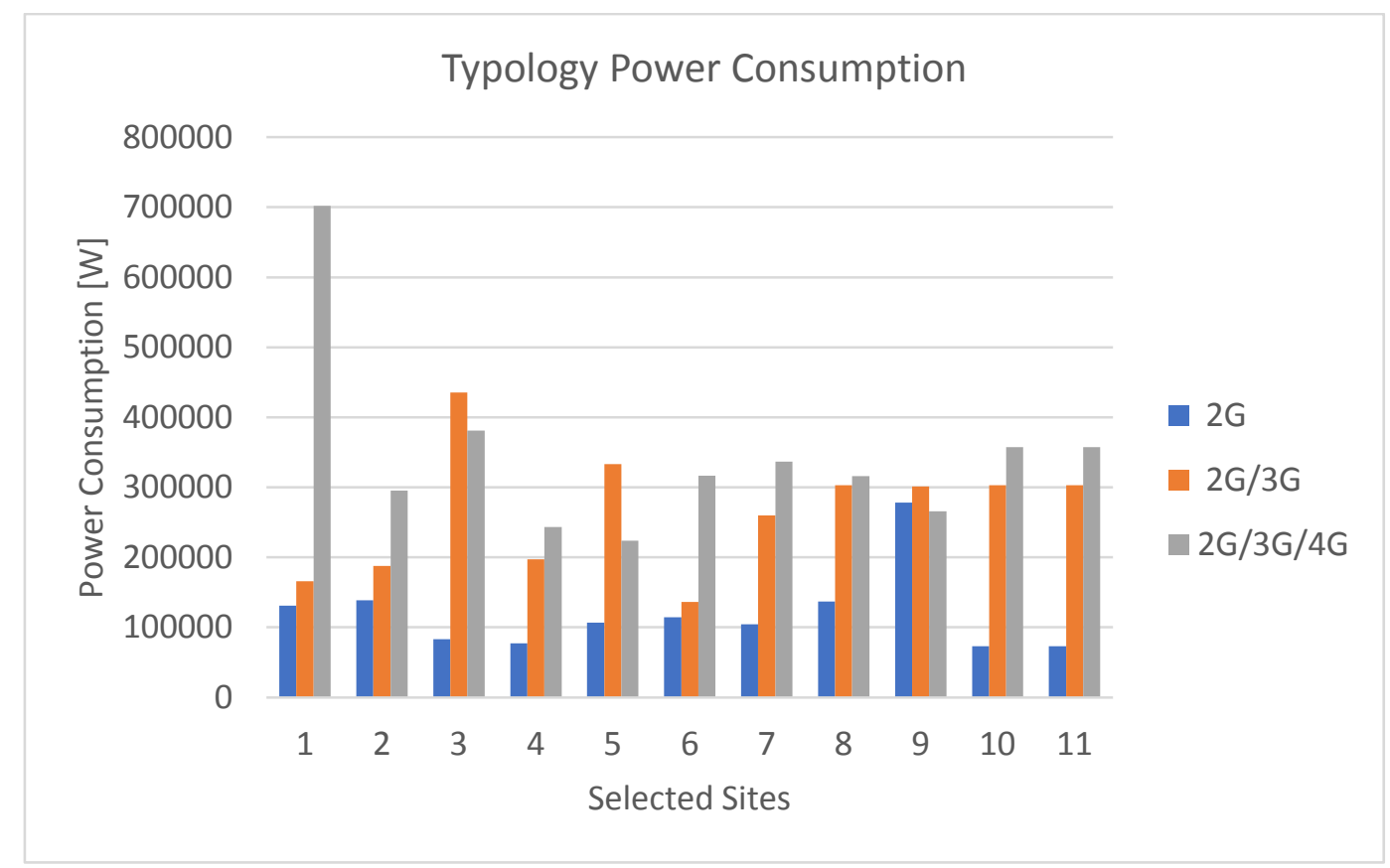

Figure 1 : Power Consumption of different typology

It is also observed in the particular case of the site 9 that the power consumption of $2 \mathrm{G}$, the GSM technology, is equally high as compared to other two typologies. This could prompt a reserved decision of the telecom operators for the necessary need to go toward the advanced technology standard.

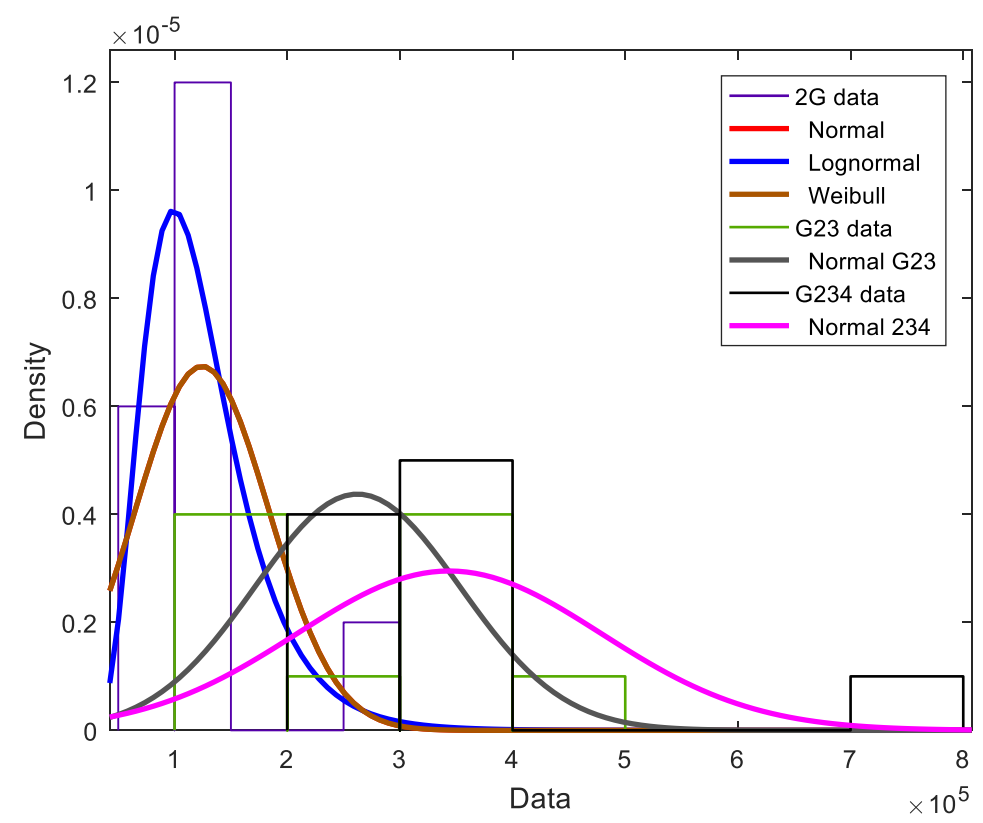

Figure 2 : Power Profile distribution

Instead the results observed (see Figure 1) on the site 1 and 5 may be quantified to be important due to the fact that the high power consumption is observed from the three typologies which in turn, the telecoms operators may not patronize.

Further analysis of the results in Figure 1, may indicate that the power consumption of the mixed typology is definitely high, however if the cost of site acquisition for each standalone typology is considered, it may be argued that the mixed typology should be commendable for the telecom operators. 
In Figure 2, it has been illustrated the power profile distribution's characteristic of the various typologies considered. The analysis indicated that the power profile could be described by the normal distribution. Thus, the normal distribution could be used to quantify the power consumption of the respective transmission nodes that were considered in the study with a monthly mean value $124 \mathrm{kWh}, 254$, kWh and $362 \mathrm{kWh}$ respectively for 2G, $2 \mathrm{G} / 3 \mathrm{G}$, and $2 \mathrm{G} / 3 \mathrm{G} / 4 \mathrm{G}$.

\section{Conclusion}

The energy efficiency for wireless communication systems is very important for the current and the future generation ones. It should be stated that the energy consumption in communication systems is constantly increasing due to the exponential number of subscribers and high data services demand.

This article presents an evaluation of the factual quantifications methods for the energy consumption of transmission nodes in cellular communications systems. The data were collected on Moov-Africa installation in Togo. These data have undergone a preprocessing in the Microsoft Excel software, then sent in the Matlab software for further analysis. The results showed that the energy consumption observed at the site is around $124 \mathrm{kWh}, 254$, $\mathrm{kWh}$ and $362 \mathrm{kWh}$ on monthly average respectively for $2 \mathrm{G}, 2 \mathrm{G} / 3 \mathrm{G}$, and $2 \mathrm{G} / 3 \mathrm{G} / 4 \mathrm{G}$ typology used. It further indicated that when more communication standards are used on a given site, its power consumption is much more increasing. The power profile distribution has been investigated, and the analysis revealed that the normal distribution closely fitted the data. However, more parameters related to the number of utilised channels and climatic conditions need to be considered in future research works.

\section{References}

[1] Gyamfuaa JM, Ofosu WK, Dotche K, Diawu K. Investigation of the User Redial and its Effect on the Network Congestion in Call Admission : A Case Study in Ghana. In: Arai K, Bhatia R, Kapoor S, eds. Advance in Artificial Intelligent Systems (Proceedings of the Future Technologies Conference (FTC) 2018). 881. Switzerland: Springer Nature; 2019:598-607. https://doi.org/https://doi.org/10.1007/978-3-030-02683-7 42

[2] Qian Li, Hu RQ, Yi Qian, Geng Wu. Cooperative communications for wireless networks: techniques and applications in LTE-advanced systems. IEEE Wireless Communications. 2012 04;19(2). https://doi.org/10.1109/mwc.2012.6189409

[3] Dotche K, Salami A, Kodjo K, Sekyere F, Bedja K. Studying the Impacts of the Renewable Energy Integration in Telecommunication Systems: A Case Study in Lome. In: Arai K, Bhatia R, Kapoor S, eds. Advances in Intelligent Systems and Computing (Proceedings of the Future Technologies Conference (FTC) 2019). 1070. Switzerland: Springer Nature ; 2020:758-780.

[4] Tombaz S, Monti P, Kun Wang, Vastberg A, Forzati M, Zander J. Impact of Backhauling Power Consumption on the Deployment of Heterogeneous Mobile Networks. 2011 IEEE Global Telecommunications Conference - GLOBECOM 2011. 2011 IEEE Global Communications Conference (GLOBECOM 2011). 2011 Dec. https://doi.org/10.1109/glocom.2011.6133999 